\title{
Assessment of the Knowledge about Routine Laboratory Procedures in Fabrication of Fixed Dental Prostheses amongst Dental Laboratories in Western Maharashtra Region
}

\author{
Aaditee Vishnu Vandeㄹ, Shivsagar Tewary², Pronob Kumar Sanyal³ ${ }^{3}$ Karuna Gajanan Pawashe ${ }^{4}$ \\ 1, 2, 3, 4 Department of Prosthodontics and Crown \& Bridge, \\ School of Dental Sciences, KIMSDU, Karad, Maharashtra, India.
}

\section{ABSTRACT}

\section{BACKGROUND}

The success of fixed dental prosthesis (FDPs) delivered to the patients depends upon the implementation of appropriate laboratory procedures and dental materials which are required during fabrication of that prosthesis. Inappropriate technique during its fabrication may not always hamper the esthetic end results or fit, but may also hamper the longevity as well. Thus, knowledge about the dental materials as well as the basic laboratory procedures among the dental laboratories should be regularly assessed to assure good quality work. This survey was conducted to assess the knowledge among laboratory technicians regarding the routinely used laboratory procedures and materials for fabrication of FDPs in dental laboratories in Western Maharashtra region with the help of a validated questionnaire.

\section{METHODS}

An electronic survey was conducted with the help of a validated questionnaire that was circulated to 49 laboratories in Western Maharashtra region. The feedback was gathered from laboratory technicians and was then statistically analysed to achieve the objectives of the study.

\section{RESULTS}

$48.8 \%$ of laboratories confirmed that technicians working for them were registered under the state council. $51 \%$ agreed that they only used dental materials that were American Dental Association (ADA) specified. The most common gypsum product for pouring of impressions was dental stone. $65.9 \%$ of the technicians agreed that they inspected the cast before starting the fabrication procedure. Die preparation and ditching procedure was done by $52.9 \%$ and $35.7 \%$ laboratories respectively. Only $38.1 \%$ agreed of using beryllium free alloy ingots; in $66.7 \%$ laboratories, ceramic work was carried out in a separate ceramic room with $76.2 \%$ having adequate ventilation provisions.

\section{CONCLUSIONS}

Response of technicians in Western Maharashtra region regarding their knowledge about basic laboratory procedures in FDP fabrication indicates several areas of weakness. Avoiding such inadequacy in laboratories can significantly reduce FDP failure rates.

\section{KEY WORDS}

Fixed Dental Prosthesis, Dental Laboratory, Survey
Corresponding Author: Dr. Aaditee Vishnu Vande, Department of Prosthodontics and Crown \& Bridge, School of Dental Sciences, KIMSDU, Karad, Maharashtra, India. E-mail:v.aaditee@gmail.com

DOI: $10.14260 / j e m d s / 2021 / 225$

How to Cite This Article: Vande AV, Tewary S, Sanyal PK, et al. Assessment of the knowledge about routine laboratory procedures in fabrication of fixed dental prostheses amongst dental laboratories in Western Maharashtra region. J Evolution Med Dent Sci 2021;10(15):1054-1059, 10.14260/jemds/2021/225

Submission 31-07-2020, Peer Review 15-02-2021, Acceptance 22-02-2021, Published 12-04-2021.

Copyright (C) 2021 Aaditee Vishnu Vande et al. This is an open access article distributed under Creative Commons Attribution License [Attribution 4.0 International (CC BY 4.0)] 


\section{BACKGROUND}

The field of prosthodontics is one of the most demanding disciplines of dentistry comprising of both clinical and laboratory procedures. When prosthetic rehabilitation of a patient is advised, there are several treatment options ranging from removable to fixed prostheses that a patient can select from. Selection of a specific treatment option for the patient largely depends upon several factors such as; age, systemic condition, economic and time constrain, condition of remaining intraoral structures etc. The improvements in public attitude and awareness towards dentistry has led to patients willingly opt for more complex and extensive treatment options than before. With this, it has become even more challenging for the dentists trying to satisfy the requirements of the patients in providing the required dental treatment. Considering comfort as one of patient's prime requirement, preference of fixed prosthesis over removable is always higher. Thus, fixed dental prostheses (FDP) has become one of the most important part in the field of prosthodontics. A fixed prosthesis provides exceptional satisfaction to both patient and dentist in treatment of missing dentition by adequately restoring form, function and aesthetics. The fabrication of high quality, durable dental prosthesis is considered a reflection of the skills of both the dental practitioner and dental technician. Several factors play a key role in success of FDP directly by the dentist such as clinical management of the patient, case selection, tooth preparations, adequate impression making, cementation and follow up. Another important aspect is carefully transferring the dental impressions and / or dental casts to the dental laboratory and providing all the detailed information required for fabrication of the required prostheses. ${ }^{1}$

Once adequate information is provided to the dental laboratory by the dentist, the skill and knowledge of the dental laboratory technicians is greatly responsible for fabrication of FDP that in turn affects the treatment outcome. Studies have emphasised the role of adequate communication and cooperation between dentists and dental laboratory technicians for any successful prosthodontic treatment. ${ }^{1}$ Apart from communication, the success of a FDP delivered to the patient depends upon the implementation of appropriate laboratory procedures during fabrication of that prosthesis. ${ }^{2}$ If improper and inadequate techniques are followed during the fabrication of any fixed prostheses, it may not only affect the aesthetic end results or fit but may also hamper their longevity. An improperly designed FDP may result in a prosthesis that has been fabricated with little or no reference to the important clinical or biological information. This in turn may potentially cause tissue damage and ultimately lead to failure of treatment. Having adequate knowledge of all the basic laboratory procedures in fabrication of FDP allows the working technicians to fabricate a prosthesis which is biologically, aesthetically and functionally appropriate. Thus, it is important to assess the knowledge regarding the basic laboratory procedures in fabrication of FDP among the dental laboratory technicians. This study intends to evaluate the knowledge of basic fabrication procedures followed by the laboratories for fabrication of FDP through the help of an electronic survey administered to the laboratory technicians in Western Maharashtra region.

\section{METHODS}

A cross sectional electronic survey was conducted to assess the knowledge among laboratory technicians regarding the routinely used laboratory procedures for fabrication of FDPs in dental laboratories in Western Maharashtra region with the help of an online questionnaire. Study duration was from February 2019 to May 2019.

The questionnaire was first piloted for validation on a small number of dental technicians available locally. It was distributed personally to laboratory technicians available locally and the results were analysed. Accordingly, the validity of the questionnaire was assessed and necessary changes were made in the questions. The final questionnaire consisted of 29 dichotomous questions covering the basic laboratory procedures in fabrication of FDPs. The electronic survey link was then distributed to 49 laboratories randomly around Western Maharashtra region through emails and messages with a 15 days' deadline to answer. The identity and information of all the laboratory technicians who participated in the survey were kept anonymous. The feedback was then gathered from laboratory technicians and was analysed to achieve the objectives of the study.

\section{Statistical Analysis}

All the gathered responses were tabulated and entered into Statistical Package for the Social Sciences (SPSS) version 10.0 software. The data was analysed, and the analysis was restricted to descriptive percentile statistics using mean values.

\section{RESULTS}

The response rate was $73.4 \%$ with 36 out of the 49 laboratories responding to the survey within the stipulated time period. The cumulative results of the survey are presented in Figures 1 to 3. $48.8 \%$ of laboratories confirmed that technicians working for them were registered under the state council. $51 \%$ agreed that they only used dental materials that were International Standards Organization / American Dental Association (ISO / ADA) specified. $48.8 \%$ of the work received by the labs was in the form of impressions. Disinfection of impressions before starting the procedures was carried by $28.6 \%$ of laboratories.

The most common gypsum product for pouring of impressions was dental stone (47.6\%), followed by die stone (28.6\%) and dental plaster (less than $15 \%) .64 \%$ of laboratories added gypsum product to water for mixing, with $40.5 \%$ of them using vacuum mix. 


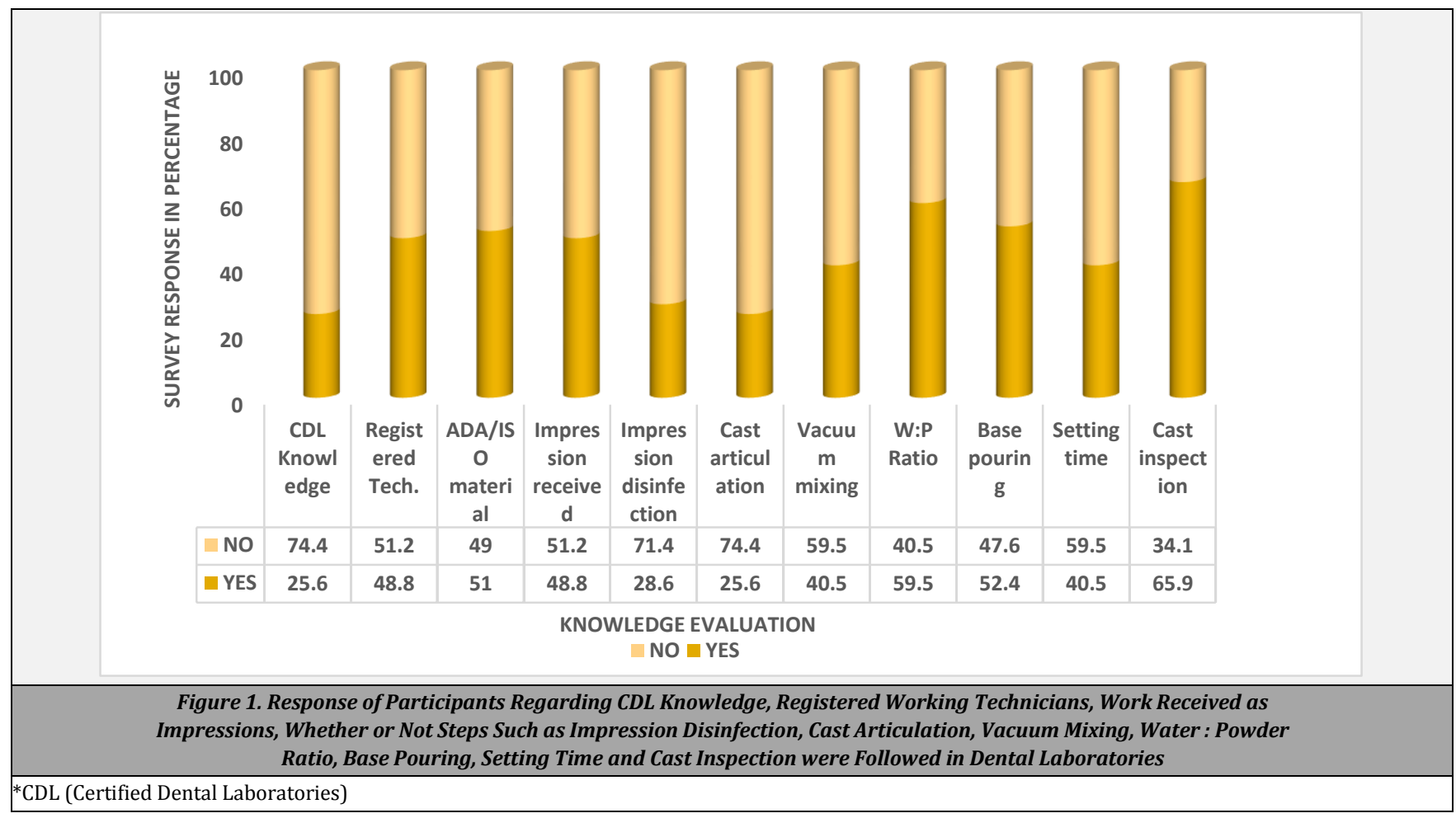

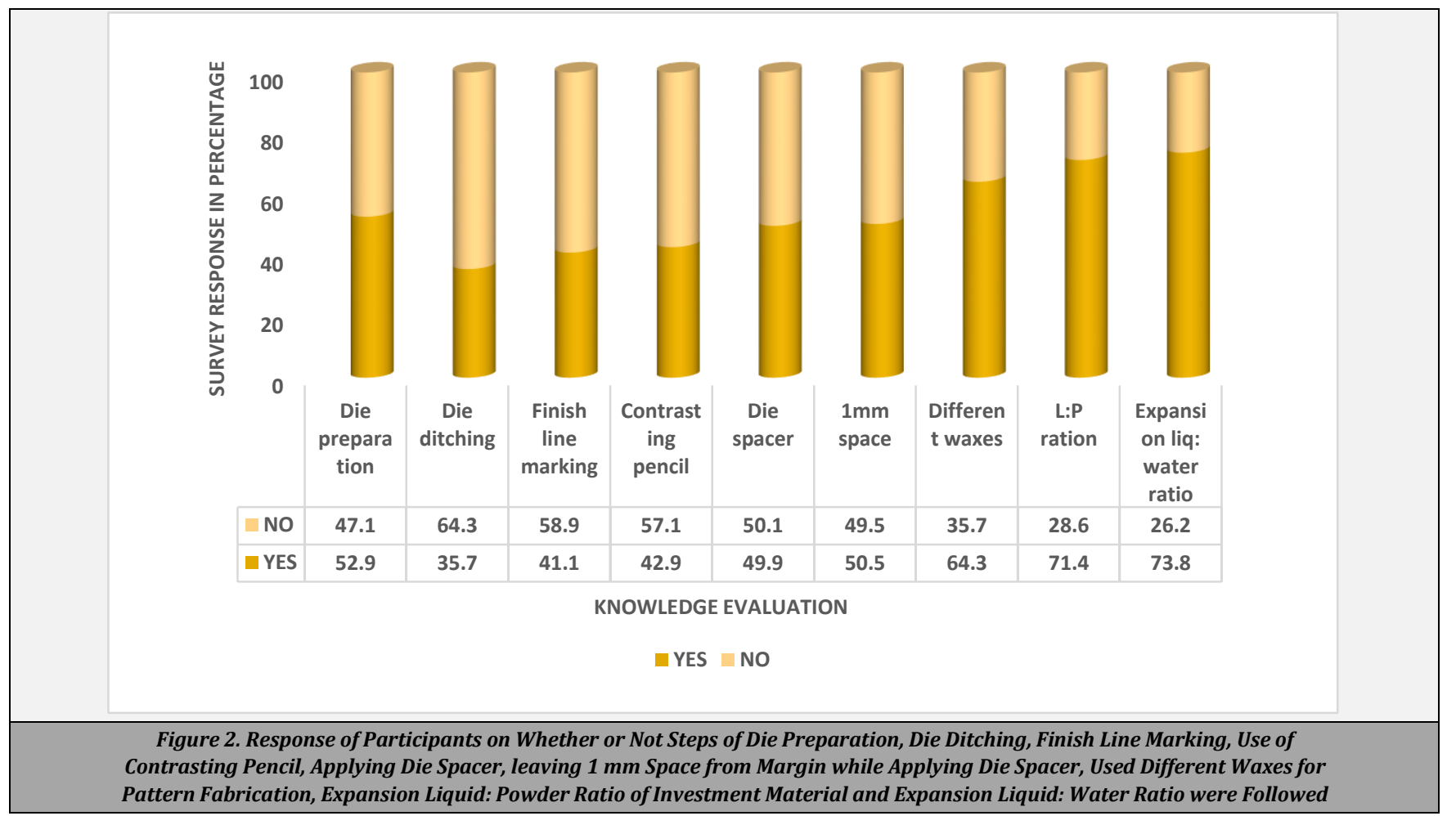

The manufacturer recommended water powder ratio was followed by $59.5 \%$ laboratories, whereas $40.5 \%$ followed the manufacturer recommended setting time. $65.9 \%$ of the technicians agreed that they inspected the cast before starting the fabrication procedure. Die preparation and ditching procedure was done by $52.9 \%$ and $35.7 \%$ laboratories respectively.

While $41.1 \%$ laboratories marked the finish line on the die, only $42.9 \%$ of them used a contrasting pencil to do the same. $49.9 \%$ of technicians used die spacer with $50.5 \%$ leaving $1 \mathrm{~mm}$ space from the margin. $64.3 \%$ used different waxes indicated while carving the wax patterns for FDPs.
Considering the investment procedure for wax patterns, 71.4 $\%$ followed the recommended liquid to powder ratio of the investment material, while $73.8 \%$ followed the expansion liquid to water ratio recommended by the manufacturer. Only $38.1 \%$ agreed of using beryllium free alloy ingots and having knowledge regarding berylliosis. In $66.7 \%$ laboratories ceramic work was carried out in a separate ceramic room, with $76.2 \%$ having adequate ventilation provisions. $61.9 \%$ practiced reuse of the sand in sand blasting unit several times. Regular ceramic firing unit calibration was done by half of the participants. 


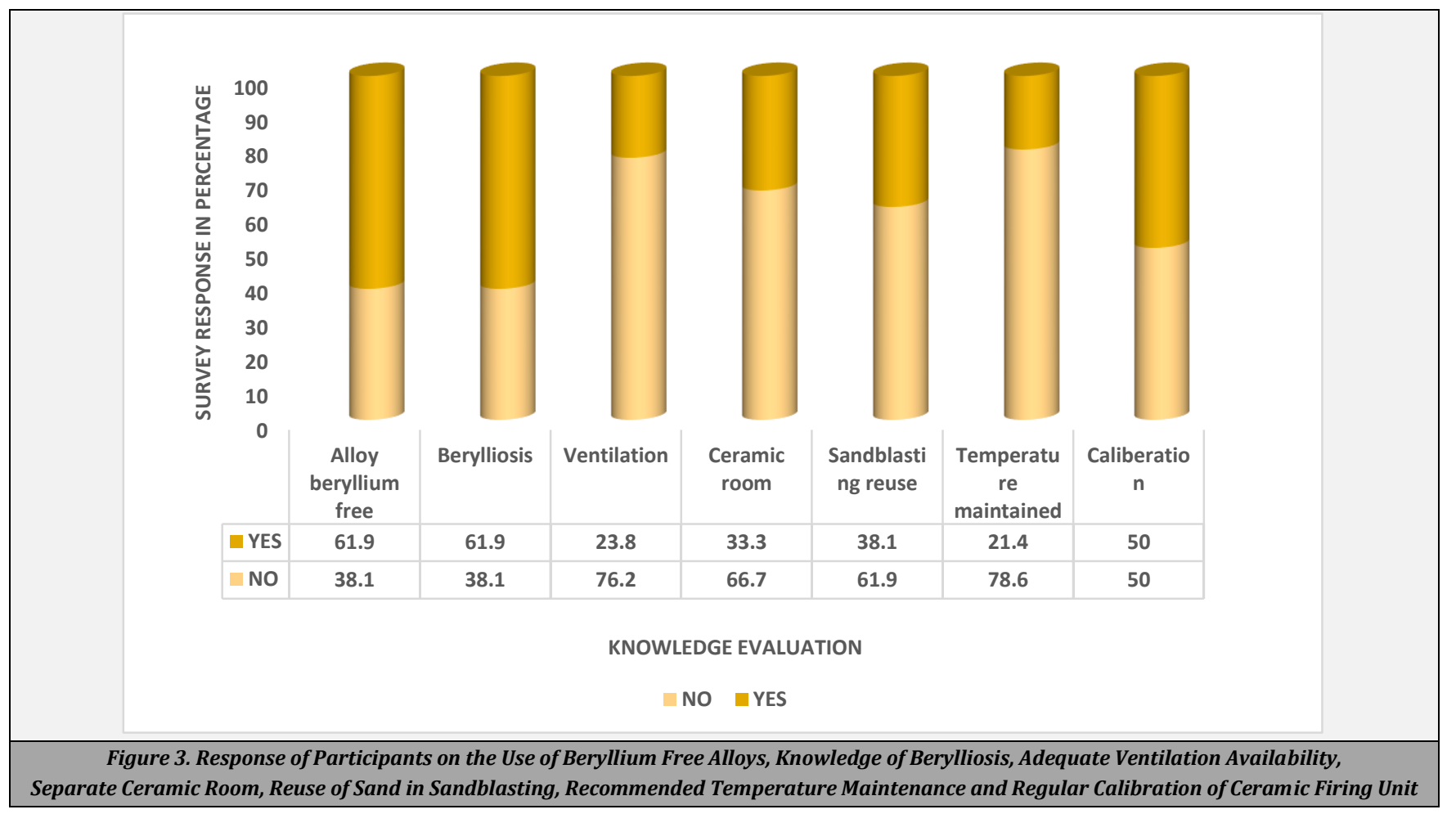

\section{DISCUSSION}

With increasing awareness and knowledge of patients regarding their own dental health, there has been a tremendous change in their attitude and responses to the treatment they receive in a dental clinic. With information about the advanced and more comfortable treatment modalities available, patient's interest has shifted towards fixed prostheses than removable ones. The ethical and legal responsibilities of both the dentist and dental laboratory technicians play a key role in successfully treating a patient. Relying only on the dentist's knowledge and authority to delegate laboratory procedures based on the functional and esthetical demands of the patient is not sufficient. The assistance provided by dental laboratory technicians in fabricating a prosthesis is equally important. Several surveys have been carried out in the past to evaluate the efficiency of communication between the dentist and lab technicians. ${ }^{2-6}$ Results of these studies have greatly improved the current communication strategies between the two for improved quality of work.

However, one of the major concerns that has not been focused upon is the use of correct techniques in fabrication of prostheses especially for fixed crowns and bridges that require great precision and fit. Improved communication in fabricating FDPs would be of no use if the final prosthesis is not satisfactory due to improper fabrication techniques. This survey study was specially designed to assess the knowledge amongst dental laboratory technicians regarding the routine dental laboratory procedures in fabrication of FDPs in western Maharashtra region.

Cumulative evaluation of the entire survey showed that a mean of $52.2 \%$ followed the correct technique and had adequate knowledge about the same. It was found that only
$48.8 \%$ were registered under state council and only a quarter of the participants were aware about the specifications provided by the Dental Council of India through the Clinical Establishment Act Standards for Dental Lab. ${ }^{7}$ Protocols regarding laboratory setup provided by them should be followed by all dental technicians as it provides highest standards of professional laboratory excellence in India. With only about $25 \%$ laboratories using articulators in fabrication of FDP shows the lack of adherence to proper technique. Hand held casts during fabrication of single or multi-unit FDPs do not provide adequate maxillomandibular relationship and might lead to improper occlusion or discomfort to the patient after final cementation. ${ }^{8}$ Another very important and commonly neglected step by laboratories is disinfection. ${ }^{9}$ Following robust disinfection protocols is of utmost importance to prevent cross-infections between the patient, dentist and lab personals.

In the present survey, $71.4 \%$ laboratories did not follow standard disinfection protocol, thereby increasing the risk of cross contamination and infection. Similar results were recorded by Kugal G et al. ${ }^{10}$ and Hatzikyriakos A et al. ${ }^{4}$ in their studies to evaluate the efficiency of dental laboratories to implement disinfection protocol for impressions. This suggests that awareness and practices of dental technicians regarding cross-infection control are less than ideal and must be improved. However, the technicians must also be aware to avoid repetitive disinfection as it poses a risk of dimensional stability changes and also hamper the surface detail reproduction. ${ }^{11}$ for this, the communication between dentists and dental technicians is essential.

Dental cast is a positive replica of the teeth and surrounding tissue. Various factors are responsible to obtain a good quality dental cast; such as the dental material used, following the manufacturer recommended water powder ratio, mixing technique, setting time, base pouring and finally 
inspecting the cast thoroughly before initiating with the fabrication procedure. In the present survey, knowledge regarding the above factors ranged between $40.5 \%$ to $65.9 \%$ with a mean value of $41.7 \%$. It is important that laboratories not only critically evaluate the dental cast that is obtained but also give importance to all the steps from pouring of cast to the final setting period. Failure to do so might lead to fabrication of a fixed prosthesis that may adequately fit on the cast but not intraorally when trying it in the patient's mouth.

Die preparation is another important step in FDP fabrication. The present survey concentrated only on whether or not the die preparation was carried out by the laboratories. About $50 \%$ responded positively to the question. Similarly, less than half of the laboratories followed steps of die ditching, marking the finish line in contrasting color and die spacer application 0.5 to $1 \mathrm{~mm}$ above the finish line. A long term FPD requires adequate fit and marginal adaptation. Avoiding the above-mentioned steps may lead to fabrication of the FPD with hampered quality. Inability of the prosthesis to sit over the prepared tooth margins and adapt to it may inhibit routine hygiene procedures and encourage dental caries and gingival inflammation. ${ }^{12}$ Even the most ideal and suitable case selected for FDP may fail in due course of time if the above laboratory steps were not followed adequately.

Risk of respiratory infections and berylliosis in lab technicians using beryllium containing alloys in dental prosthesis fabrication have been reported earlier. ${ }^{13-15}$ Since then, there has been a continuous emphasis on use of beryllium free alloys in dental practice. The present survey recorded $38.1 \%$ laboratories using beryllium free alloy in their laboratories and the same had knowledge about berylliosis. This result shows the lack of knowledge and awareness amongst dental technicians towards risk factors related to their own health. Other important aspect covered in the survey were whether or not having a separate ceramic room with proper temperature control and ventilation. Also, whether the laboratories were reusing the sand in sand blasting unit and regularly calibrating the ceramic firing unit. All these questions had a similar response pattern. The overall analysis of the results of this survey suggest that approximately half of the laboratories who participated in the study were not following the routine laboratory procedures and steps required in the fabrication of FDP. This can be considered as one of the major causes of increased failure rate of fixed dental prostheses in a short duration of time. Working with more caution would largely reduce the FDP failure rate in future.

\section{CONCLUSIONS}

Within the limitations of this survey study, it can be identified that there is inadequacy of knowledge about the basic laboratory procedures used in fabrication of FDPS amongst the lab technicians. Emphasis should be given on use of appropriate procedures in fabrication of FDPs in dental laboratories so as to improve the quality of work delivered by the laboratories. Evaluating responses given by the dental laboratory technicians in Western Maharashtra region in the survey regarding their knowledge about basic laboratory procedures in FDP fabrication, highlights their areas of weakness. Avoiding such inadequacy in laboratories can significantly reduce FDP failure rates. Also, conducting workshops and continuing dental education programs for the dental laboratory technicians will help in enhancing their knowledge and even acquaint them with the newer techniques and advances in dental laboratory procedures.

Data sharing statement provided by the authors is available with the full text of this article at jemds.com.

Financial or other competing interests: None.

Disclosure forms provided by the authors are available with the full text of this article at jemds.com.

The authors would like to acknowledge all the dental laboratory technicians who participated in the study.

\section{REFERENCES}

[1] Afsharzand Z, Rashedi B, Petropoulos VC. Communication between the dental laboratory technician and dentist: work authorization for fixed partial dentures. J Prosthodont 2006;15(2):123-8.

[2] Jenkins SJ, Lynch CD, Sloan AJ, et al. Quality of prescription and fabrication of single-unit crowns by general dental practitioners in Wales. J Oral Rehabil 2009;36(2):150-6.

[3] Leith R, Lowry L, O'Sullivan M. Communication between dentists and laboratory technicians. J Ir Dent Assoc 2000;46(1):5-10.

[4] Hatzikyriakos A, Petridis HP, Tsiggos N, et al. Considerations for services from dental technicians in fabrication of fixed prostheses: a survey of commercial dental laboratories in Thessaloniki, Greece. J Prosthet Dent 2006;96(5):362-6.

[5] Aquilino SA, Taylor TD. Prosthodontic laboratory and curriculum survey. Part III: fixed prosthodontic laboratory survey. J Prosthet Dent 1984;52(6):879-85.

[6] Stewart CA. An audit of dental prescriptions between clinics and dental laboratories. Br Dent J 2011;211(3):E5.

[7] Clinical Establishment Act. Standard For Dental Lab Standard No.CEA / Dental Lab. http://clinicalestablishments.gov.in/WriteReadData/31 91.pdf

[8] Steele JG, Nohl S, Wassell RW. Crowns and extra-coronal restorations: occlusal considerations and articulator selection. Br Dent J 2002;192(7):377-80.

[9] Almortadi N, Chadwick RG. Disinfection of dental impressions-compliance to accepted standards. Br Dent J 2010;209(12):607-11.

[10] Kugel G, Perry RD, Ferrari M, et al. Disinfection and communication practices: a survey of $U$ S. dental laboratories. J Am Dent Assoc 2000;131(6):786-92.

[11] British Dental Association. Advice sheet A12, infection control in dentistry. London: BDA 2009.

[12] Libby G, Arcuri MR, LaVelle WE, et al. Longevity of fixed partial dentures. J Prosthet Dent 1997;78(2):127-31.

[13] Hugonnaud C, Lob M. Risks encountered by dental technicians during manufacture of metallic prostheses. Med Soc Prev 1976;21(4):139.

[14] Hinmann RV, Lynde TA, Pelleu CB, et al. Factors affecting airborne beryllium concentrations in dental spaces. J Prosthet Dent 1975;33(2):210-5. 
[15] Fireman E, Kramer MR, Priel I, et al. Chronic beryllium disease among dental technicians in Israel. Sarcoidosis Vasc Diffuse Lung Dis 2006;23(3):215-21. 